\title{
Screening for undiagnosed obstructive airway disease: Does it make sense?
}

\author{
Shawn Aaron MDCM MSc FRCPC
}

$\mathrm{T}$ he article by Robitaille et al (1) in the current issue of the Journal (pages 209-214) details results of the investigators' efforts to undertake targeted screening for obstructive lung disease in patients attending a routine preoperative assessment clinic at The Jewish General Hospital in Montreal, Quebec. The investigators used prebronchodilator spirometry to target all previous or current smokers in an attempt to detect obstructive lung disease. As expected, $41 \%$ of this 'at-risk' cohort had spirometric evidence of airflow obstruction. Many of the patients identified as having obstructive lung disease in this clinic had never been previously diagnosed with chronic obstructive pulmonary disease or asthma.

This important study supports results generated by Canadian epidemiological researchers suggesting that most individuals with airflow obstruction in Canada remain undiagnosed. The 2013 Canadian Health Measures Survey (2) found that $11 \%$ of the Canadian population 35 to 79 years of age had airflow obstruction; however, $90 \%$ of these subjects were undiagnosed. Similarly, the COLD study (3), a population-based study, screened 4893 adult Canadian subjects and found that $10.5 \%$ had undiagnosed airflow obstruction. Results from both studies suggest that $10 \%$ to $11 \%$ of the general Canadian adult population have undiagnosed airflow obstruction. Clearly, the asthma and chronic obstructive pulmonary disease we physicians see in our clinical practices may be the tip of the iceberg.

Unfortunately, one problem with the screening studies performed to date is that they identify a potential public health problem undiagnosed airflow obstruction is relatively common in Canadians but they do not follow up and tell us how important this problem is to affected individuals and to the health care system. Most undiagnosed airflow obstruction detected in the community is relatively mild and, theoretically, this degree of lung function impairment may not be clinically important. Unfortunately, the burden of undiagnosed disease on the patients and on the health care system, remains unstudied and, hence, unknown.

The other obvious problem with screening for undiagnosed airway disease is that until a therapeutically effective strategy can be tied to screening to impact patient outcomes, screening programs may be of limited clinical relevance. Why screen for early diagnosis of a disease if making the diagnosis will not improve patient outcomes or provide benefit to the Canadian health care system?

What's the next step? We need Canadian screening studies that identify patients with undiagnosed obstructive lung disease from our communities and assess the subsequent burden of lung disease in these individuals using validated symptom scores and measurements of health-related quality of life as well as health care utilization. We need Canadian clinical trials that target these newly diagnosed individuals with guideline-directed interventions including smoking cessation, immunizations, education and appropriate bronchodilators and/or anti-inflammatory therapy for airflow obstruction. The goal would be to determine whether screening for obstructive lung disease coupled to a comprehensive evidence-based treatment strategy will improve health outcomes for patients and be cost effective.

Are we getting there? Yes! The Canadian Respiratory Research Network (CRRN) will be starting a study shortly to answer the these questions. Hopefully, the CRRN study - and others like it - will let us know whether widespread screening studies of at-risk populations should be implemented to look for undiagnosed airway disease in our communities.

\section{REFERENCES}

1. Robitaille C, Dajczman E, Hirsch A, et al. Implementation of a targeted screening program to detect airflow obstruction suggestive of chronic obstructive pulmonary disease within a presurgical screening clinic. Can Respir J 2015;22:209-14.

2. Canadian Health Measures Survey: Household and physical measures data, 2012 to 2013. <www.statcan.gc.ca/dailyquotidien/141029/dq141029c-eng.htm> (Accessed January 26, 2015).

3. Tan WC, Bourbeau J, Fitzgerald JM et al. Can age and sex explain the variation in COPD rates across large urban cities? Int J Tuberc Lung Dis 2011;15:1691-8. 


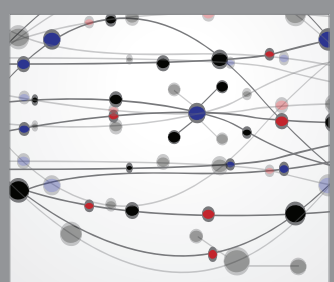

The Scientific World Journal
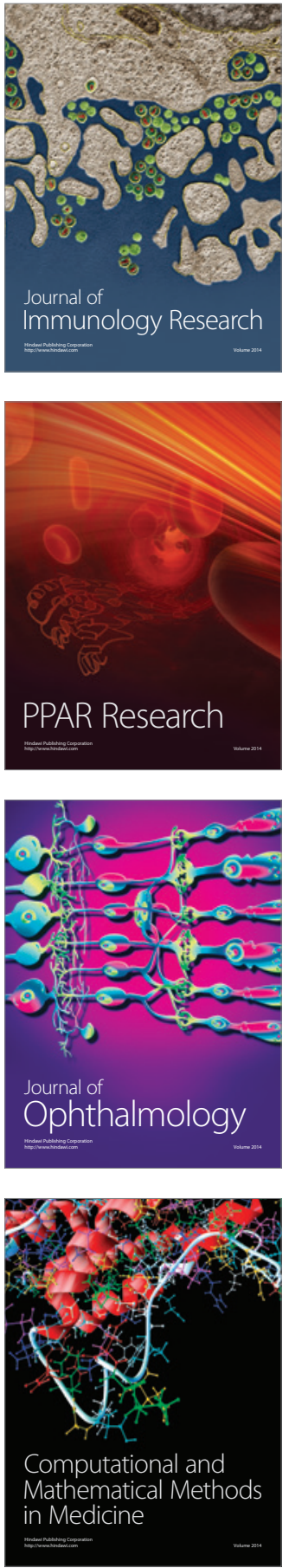

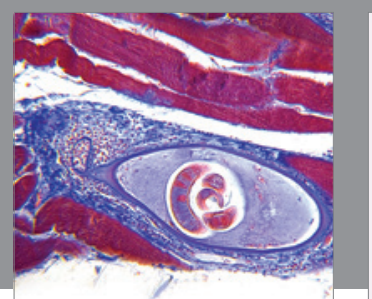

Gastroenterology Research and Practice

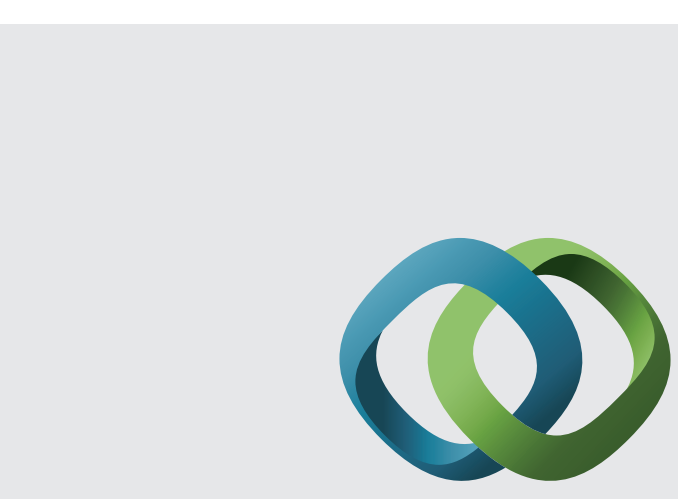

\section{Hindawi}

Submit your manuscripts at

http://www.hindawi.com
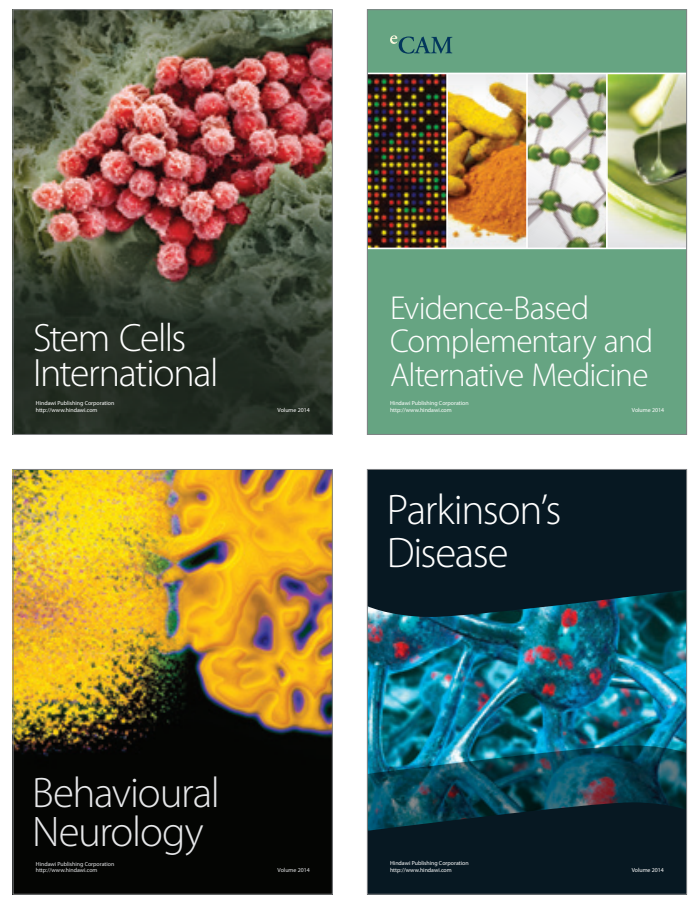
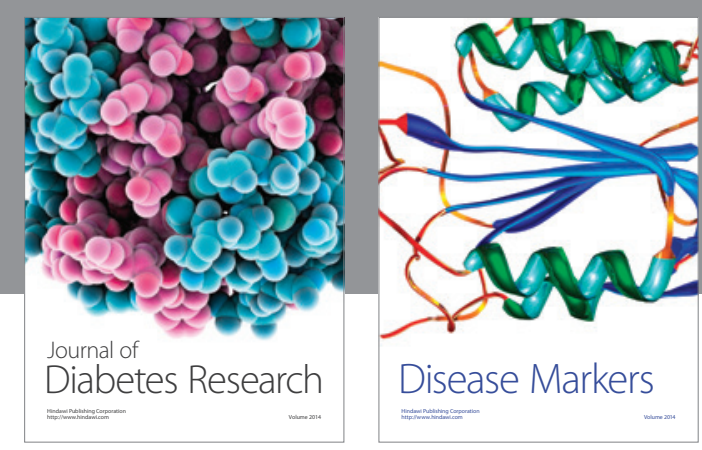

Disease Markers
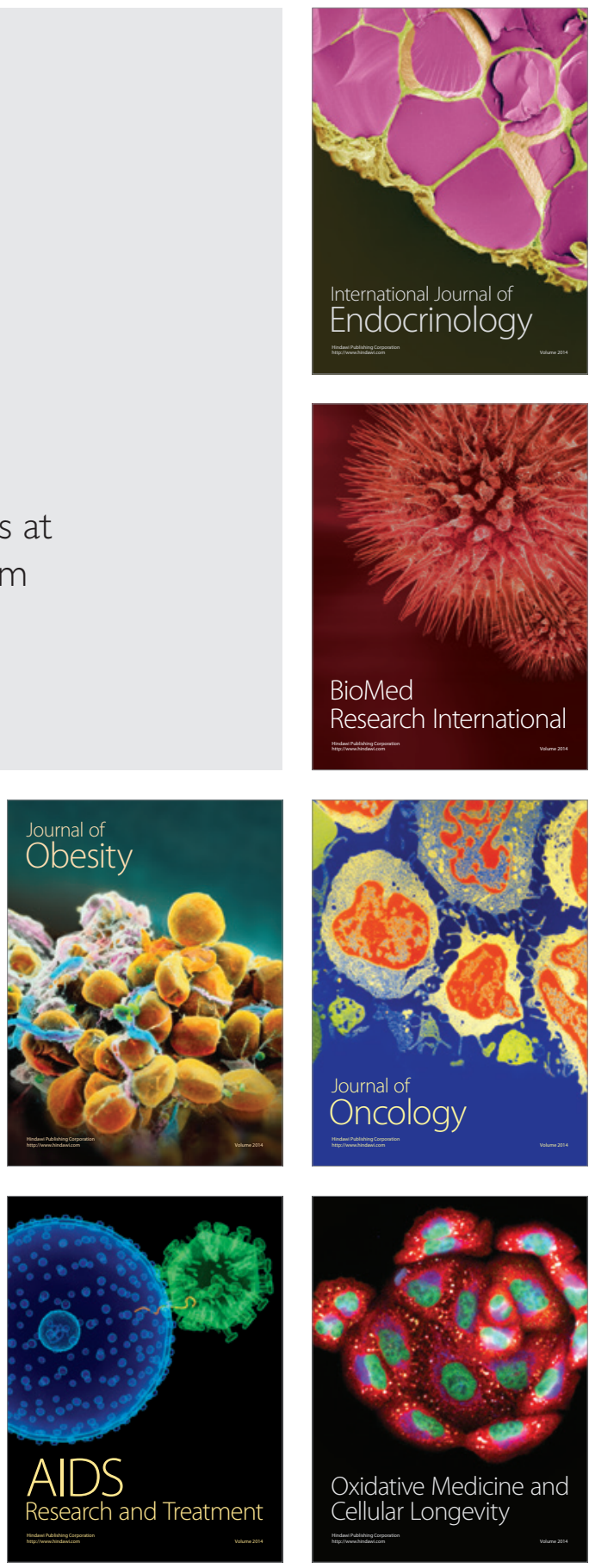